\title{
Budding Response of Horticultural Crops to Night Break with Red Light on Alternate Days
}

\author{
Hiroshi Hamamoto*, Hideo ShimaJI ${ }^{* *}$ and Tadahisa Higashide*** \\ National Research Institute of Vegetables, Ornamental Plants and Tea, Taketoyo, Aichi 470-2351, Japan
}

(Received September 17, 2004)

\begin{abstract}
To investigate whether night break (i.e., the interruption of the dark period by artificial lighting) controls plant flowering sufficiently with treatment on alternate days, we compared the reproductive responses of seven horticultural crop species under a daily night-break treatment, an alternate-day treatment, and no night-break treatment during the short-daylength season. Longday crops of spinach (Spinacia oleracea), petunia (Petunia $\times$ hybrida), and godetia (Godetia cvs.) and short-day crops of cosmos (Cosmos bipinnatus), perilla (Perilla ocymoides), zinnia (Zinnia elegans), and Japanese morning glory (Pharbitis nil) were used. The night-break treatment was supplied for 2 hours starting at midnight using red light-emitting diodes. The effect of the alternate-day treatment was much smaller than the effect of the daily treatment in zinnia and Japanese morning glory; was smaller in spinach, godetia and cosmos; and was only slightly smaller in petunia. Budding in perilla was inhibited by both night-break treatments.
\end{abstract}

Keywords : alternate days, budding, horticultural crops, night break, response to day length

\section{INTRODUCTION}

Night break (i.e., the interruption of the natural dark period by artificial lighting) during the short-daylength season is effective in accelerating the flowering of long-day crops to permit earlier marketing or seed production, and in delaying the flowering of short-day crops to delay marketing or extend the harvesting time of leaf vegetables that set bud in response to short daylength. A night-break treatment also affects the reproductive response of short-day and long-day crops in a manner similar to that of naturally long daylength.

If night-break treatment on alternate days can control plant flowering sufficiently, electricity usage can be reduced compared with daily treatments. However, there have been few reports on plant reproductive responses to exposure to alternating night-break treatments and natural short days. Sato (1981) reported that a daily night-break treatment over 2 to 5 days following 3 or 4 naturally short days delayed chrysanthemum flowering, but did not study the effects of an alternateday night-break treatment. A few early studies reported suppression of plant flowering in response to shortened daylength created by covering the plants with a light-proof canopy on alternate days during the long-daylength season; these included a study of chrysanthemum (Post, 1934), and a study of soybean, yellow cosmos, perilla, and garden balsam (Garner and Allard, 1931).

Corresponding author : Hiroshi Hamamoto, fax: +81-773-42-7161, e-mail: ham@affrc.go.jp

Present address: * National Agricultural Research Center for Western Region, 200-Ueno, Ueno, Ayabe, Kyoto 623-0035, Japan

** National Institute of Floricultural Science, Tsukuba, Ibaraki 305-8519, Japan

*** National Agricultural Research Center for Western Region, Zentsuji, Kagawa 765-8508, Japan 


\section{H. HAMAMOTO ET AL.}

In the present study, we investigated whether a night-break treatment on alternate days could control plant flowering sufficiently by comparing the reproductive responses of seven horticultural crop species to three treatments: daily night break, night break on alternate days, and no night break.

\section{MATERIALS AND METHODS}

\section{Plants and night-break treatments}

We used three species of long-day crops - spinach (Spinacia oleracea L. 'Alrite'; Chenopodiaceae), petunia (Petunia $\times$ hybrida Vilm. 'Baccara Red'; Solanaceae), and godetia (Godetia cvs. 'Satin Deep Rose'; Oenotheraceae) - and four species of short-day crops - cosmos (Cosmos bipinnatus Cav. 'Radians Special'; Asteraceae), perilla (Perilla ocymoides L. 'Aochirimensiso'; Lamiaceae), zinnia (Zinnia elegans Jacq. 'Peter Pan Golden’; Asteraceae), and Japanese morning glory (Pharbitis nil Choisy 'Sunsmile Violet'; Convolvulaceae). Budding or bolting of these crops responds strongly to night break using red light (Hamamoto et. al., 2003a).

All of the plants were planted in 780-mL plastic pots (one plant per pot) filled with fertilized

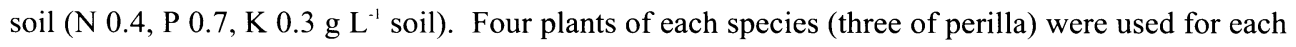
treatment. The plants in a given treatment were grown under a steel frame $600 \mathrm{~mm}$ long $\times 400 \mathrm{~mm}$ wide $\times 730 \mathrm{~mm}$ high with a ceiling. Three such frames were set up in a glasshouse to permit three treatments: a control treatment without any night break, and two night-break treatments with irradiation from 2300 to $0100 \mathrm{~h}$, either daily or on alternate days. The greenhouse was heated when the temperature fell below $13^{\circ} \mathrm{C}$ and ventilated when the temperature rose above $30^{\circ} \mathrm{C}$. Light was provided by 350 - $\mathrm{mm} \times 400$-mm panels, each consisting of $800(25 \times 32)$ red light-emitting diodes (LEDs) with peak photon emission at a wavelength of $655 \mathrm{~nm}$. One LED panel was mounted on the ceiling of the steel frame used for the night-break treatments. Before the experiment, the photon flux density from each LED panel was measured with a spectroradiometer (LI-1800, Li-Cor Inc., Lincoln, NE, USA), then adjusted to $8 \mu \mathrm{mol} \mathrm{m}^{-2} \mathrm{~s}^{-1}$ at floor level, below the center of each LED panel, by controlling the electric current supplied to the LEDs. The sides and back of each frame were covered with shadecloth from 1700 to $0900 \mathrm{~h}$ daily during the experiment so that the plants would not be affected by the other treatments.

\section{Experiment 1}

The night-break treatment began on 9 November 2000. Three species were used: spinach 4 to $6 \mathrm{~cm}$ long and with two leaves, cosmos 7 to $9 \mathrm{~cm}$ high and with four leaves, and perilla 1 to 2 $\mathrm{cm}$ high and with a pair of cotyledons. To avoid differentiation of flower buds in the short-day crops (cosmos and perilla) before the night-break treatment began, these crops were subjected to a long-day treatment by means of irradiation with an incandescent lamp between 1700 and $2100 \mathrm{~h}$ daily, from germination until the beginning of the night-break treatment.

\section{Experiment 2}

Four species were used: zinnia 3 to $4 \mathrm{~cm}$ high and with two leaves, Japanese morning glory 1 to $2 \mathrm{~cm}$ high and with a pair of cotyledons, petunia less than $1 \mathrm{~cm}$ high and with six or seven leaves, and godetia 2 to $3 \mathrm{~cm}$ high and with four leaves. The short-day zinnia and Japanese morning glory were also grown under long-day conditions as in Experiment 1 until the night-break treatment began. The night-break treatment started on 22 December 2000 for zinnia, 29 December for Japanese morning glory, 10 January 2001 for petunia, and 24 January for godetia.

\section{RESULTS}

\section{Experiment 1}

The stem (flower stalk) elongation of spinach is shown in Fig. 1a. Bolting of spinach under 
the alternate-day treatment occurred later than under the daily treatment. The stems of the spinach plants under the alternate-day treatment had not yet reached a length of $3 \mathrm{~cm}$ by 19 December (day 40 of the treatment), whereas the stems under the daily treatment had grown to nearly $40 \mathrm{~cm}$ by this time. No control plant had a visible flower stalk by day 40 .
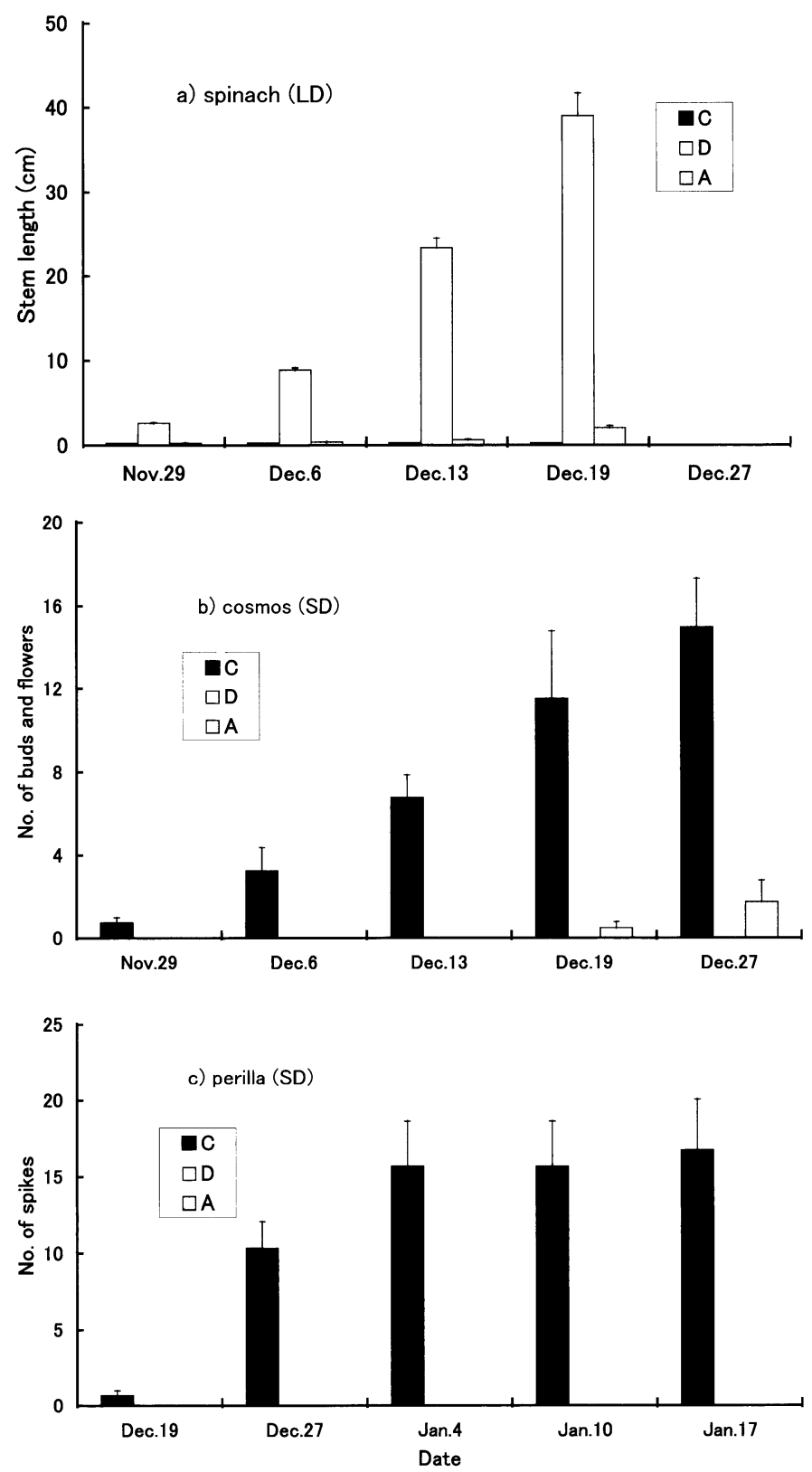

Fig. 1 Night break effect on (a) stem length in spinach, (b) number of buds and flowers in cosmos, and (c) number of spikes in perilla. Long-day and short-day crops are indicated by LD and SD, respectively. $\mathrm{C}$, control treatment without night break; D, daily night-break treatment with red LED light; A, nightbreak treatment on alternate days with red LED light. Vertical bars indicate standard errors $(n=4$ for spinach and cosmos, $n=3$ for perilla). 


\section{H. HAMAMOTO ET AL.}

The numbers of flower buds and open flowers of cosmos are shown in Fig. 1b. Flower buds had appeared in the control plants by 29 November (day 20 of the treatment), at the 8- to 12-leaf stage. Under the alternate-day treatment, flower buds first appeared on 19 December (day 40), when the plants had 15 or 16 leaves. Under daily treatment, plants had no flower buds by 27 December (day 48), when they were at the 16- to 18-leaf stage. On 27 December, open flowers were found only in the control plants.

The number of spikes in perilla is shown in Fig. 1c. The first spikes had appeared on the control plants by 19 December (day 40 of the treatment), when the plants had 8 to 10 leaves. Both the daily and the alternate-day treatments inhibited the appearance of spikes. By 17 January (day 69), there were still no spikes on any of the plants exposed to either night-break treatment, though all of the treated plants had reached the 14- to 16-leaf stage by that time.

\section{Experiment 2}

Terminal flower buds had appeared on all zinnia plants by late January. However, the appearance of the lateral flower buds was affected by the treatments. Fig. 2a presents the numbers of buds and flowers in the lateral shoots of zinnia plants. The lateral flowers appeared earliest in the control plants. Under the alternate-day treatment, lateral flower buds appeared slightly later than in the controls, but much earlier than under the daily treatment. The lateral flowers opened earlier in the control group than in the alternate-day treatment group. No open lateral flowers were apparent in the daily treatment group by 1 March (day 69 of the treatment), when the examination was ended for zinnia.

The number of flower buds in Japanese morning glory is shown in Fig. 2b. Some flower buds had appeared under the control and alternate-day treatments by 28 February (day 61 of the treatment), when the plants had reached the 6- to 7-leaf stage. Flower buds were found in all of the control and alternate-day plants by 7 March (day 68), when the plants had reached the 8- or 9-leaf stage. Flower buds appeared slightly earlier in the control group than under alternate-day treatment. All of the control plants, but only one of the alternate-day plants, had 10 or more flower buds on 20 March (day 81). Under the daily treatment, flower buds had appeared in two plants by 20 March (day 81), when the plants had 11 leaves. The remaining two plants under the daily treatment had no flower buds at that time.

In petunia (Fig. 2c), flower buds appeared earliest under the daily treatment. The first flower bud appeared in these plants at the 19-leaf stage on 14 February (day 35 of treatment). Under the alternate-day treatment, flower bud appearance progressed slightly later than under the daily treatment. In the control group, flower buds appeared later and opened later than in either night-break treatment.

In godetia, flower buds were found in all plants under the daily treatment by 28 February (day 35 of the treatment) and in one plant under the alternate-day treatment; these plants were at the 21 to 24-leaf stage. Flower buds appeared sooner and numbers increased earlier under the daily treatment than under the alternate-day treatment (Fig. 2d). In the control group, flower buds first appeared on 23 March (day 58 of the treatment, at the 43- to 48-leaf stage). On that day, there were considerably fewer flower buds in the controls than in either of the night-break treatments.

\section{DISCUSSION}

Because these experiments were carried out during the short-daylength season, budding or bolting in the long-day crops (spinach, petunia, and godetia) did not occur, or occurred late, whereas budding in the short-day crops (cosmos, perilla, zinnia, and Japanese morning glory) occurred early as a result of the naturally short daylength. Conversely, daily night-break treatment promoted flower formation in the long-day crops but not in the short-day crops. The alternate-day treatments produced three different types of effect on the plants: 

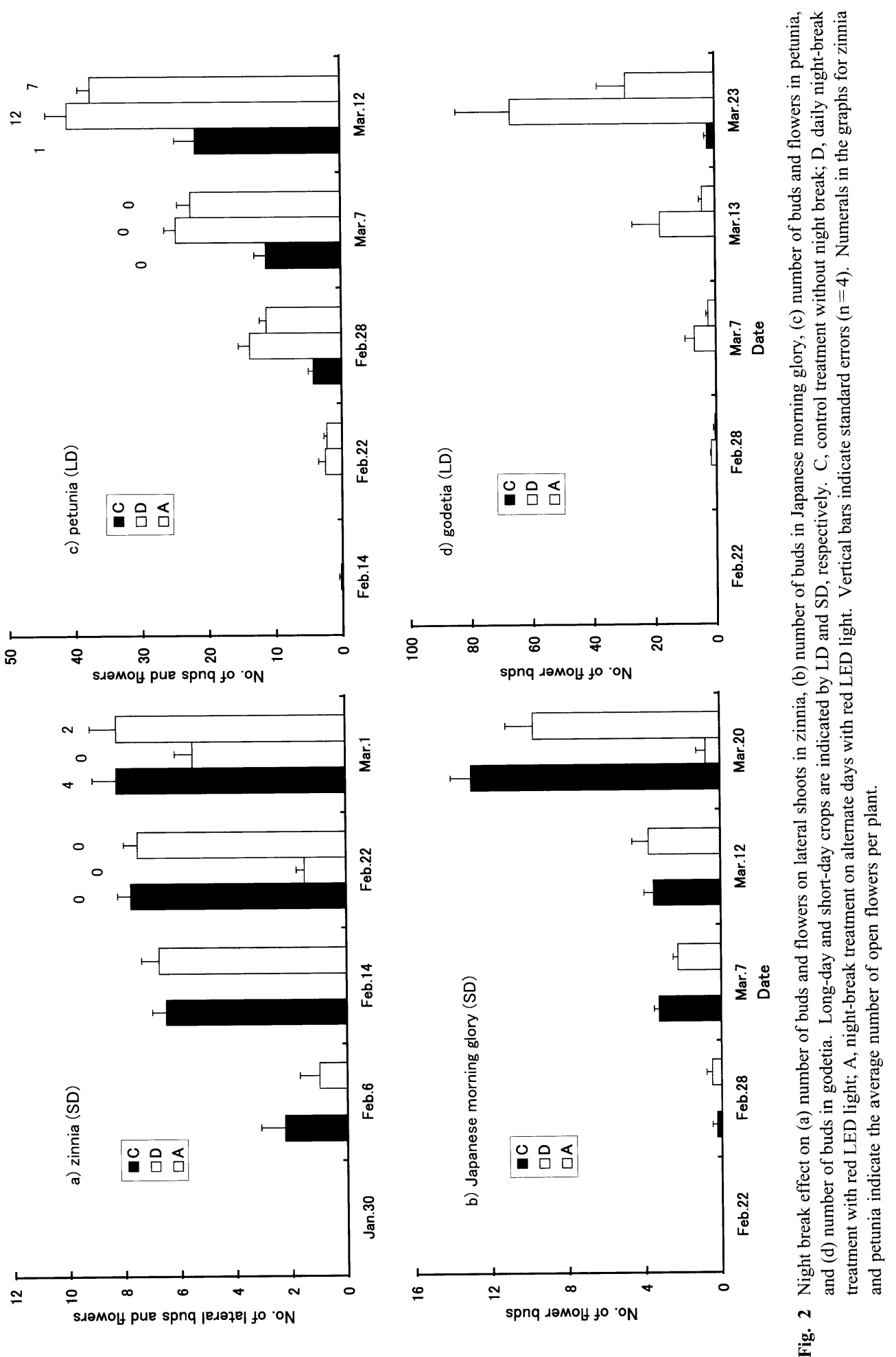


\section{H. HAMAMOTO ET AL.}

(1) The effects of alternate-day treatment were much smaller than those of the daily treatment: In zinnia and Japanese morning glory, both short-day crops, the alternate-day treatment slightly inhibited flower appearance compared with the control treatment. This inhibitory effect was very small in comparison with that of the daily treatment.

(2) The effects of the alternate-day treatment were similar to, or slightly smaller than, those of the daily treatment:

Budding by perilla, a short-day crop, was inhibited by both the alternate-day and the daily treatments. In contrast, flower formation by petunia, a long-day crop, was promoted by both the daily and the alternate-day treatments, although the effect was slightly greater with daily treatment.

(3) The effects of the alternate-day treatment differed moderately from those of the control or the daily treatment:

Unlike the control treatment, alternate-day treatment promoted bolting and budding in spinach and godetia, which are both long-day plants, but this effect was considerably smaller than the effect of daily treatment. Budding in cosmos, a short-day crop, was found under the alternateday treatment but not under the daily treatment, and was greatly inhibited by the alternate-day treatment in comparison with the controls.

Alternate-day treatment during the short-daylength season caused budding in all species except perilla. In many cases in this study, the night-break effect was shown under alternate-day treatment, but the effect was smaller than that observed under daily treatment. The alternate-day treatment would thus be impractical for controlling flowering of zinnia and Japanese morning glory, because its effects on the flowering of these plants did not differ significantly from the results for the control. In contrast, the small positive effect of alternate-day treatment on spinach bolting would be useful for winter spinach cultivation. Night-break treatment has been shown to promote both spinach growth and bolting before harvest (Narimatsu, 1996). Accordingly, Hamamoto et al. (2003b) applied night break three times per week at intervals of 1 or 2 days and were able to promote spinach growth without leading to premature bolting before harvest.

Reports by Garner and Allard (1931) and by Post (1934) suggested that changing daylength on alternate days can effectively control flowering, but that the effects are inferior to those produced by daily treatments. Garner and Allard (1931) reported that the flowering of soybean, cosmos, and perilla (short-day crops) occurred sooner and the flowering of garden balsam (a long-day crop) occurred later under a regime of full summer day-lengths alternating with 10-h days than under constant full summer daylength. Post (1934) reported that bud appearance in chrysanthemum occurred soonest with continuous short days supplemented by shading, followed by alternation between natural long days and short days, and occurred latest with natural long days. Budding of chrysanthemum was also accelerated when a short-day treatment was supplied frequently or continuously (Post, 1934).

On the basis of these reports and our results in the present study, we believe that daily nightbreak treatment during the short-daylength season would be more effective than alternate-day night-break treatment in both long-day and short-day horticultural crops. Alternate-day treatment for the control of flowering of perilla is worthy of further study, but a daily night-break treatment would be more effective for controlling flowering in the other crop species that we studied.

\section{REFERENCES}

Garner, W. W., Allard, H. A. 1931. Effect of abnormally long and short alternations of light and darkness on growth and development of plants. J. Agric. Res. 42: 629-651.

Hamamoto, H., Shimaji, H., Higashide, T. 2003a. Budding and bolting responses of horticultural plants to night-break treatments with LEDs of various colors. J. Agric. Meteorol. 59: 103-110. 
Hamamoto, H., Shimazu, T., Ikeda, T. 2003b. Effects of light break treatment every second or third night on the growth of certain leaf vegetables. Hort. Res. Jpn. 2: 307-310 (in Japanese, with English summary).

Narimatsu, J. 1996. Studies on the supplemental lighting culture of spinach (Spinacia oleracea L.). Bull. Agric. Res. Inst. Kanagawa Pref. 137: 17-23 (in Japanese, with English summary).

Post, K. 1934. Production of early blooms of chrysanthemums by the use of black cloth to reduce the length of day. Cornell Agric. Exp. Stn. Bull. 594: 3-30.

Sato, Y. 1981. The effect of the saidenshou treatment in the natural short day on the retardation of flowering and the quality of cut flower of small flowered winter flowering chrysanthemum in light culture. Bull. Kagawa Agric. Exp. Stn. 33: 29-35 (in Japanese, with English summary). 